 \\ MOUNTaiNS \\ Mathematical Publications
}

\section{DISCRETE-TIME COHEN-GROSSBERG NEURAL NETWORKS WITH TRANSMISSION DELAYS AND IMPULSES}

\author{
Sannay Mohamad — Haydar AkÇa - Valéry Covachev
}

\begin{abstract}
A discrete-time analogue is formulated for an impulsive Cohen-Grossberg neural network with transmission delay in a manner in which the global exponential stability characterisitics of a unique equilibrium point of the network are preserved. The formulation is based on extending the existing semidiscretization method that has been implemented for computer simulations of neural networks with linear stabilizing feedback terms. The exponential convergence in the $p$-norm of the analogue towards the unique equilibrium point is analysed by exploiting an appropriate Lyapunov sequence and properties of an $M$-matrix. The main result yields a Lyapunov exponent that involves the magnitude and frequency of the impulses. One can use the result for deriving the exponential stability of non-impulsive discrete-time neural networks, and also for simulating the exponential stability of impulsive and non-impulsive continuous-time networks.
\end{abstract}

\section{Introduction}

Numerical (or computer) simulations of continuous-time neural networks governed by differential equations involving transmission delays (i.e., discrete or fixed delays, time-varying delays and distributed delays) have been developed steadily over the years [14]-[21], [23]-334. These simulations are produced by discrete-time analogues formulated from semi-discretization method, in a manner in which the dynamical characterisitcs - such as the sets of equilibria and their stability characteristics - of the networks are preserved. The successful implementation of the analogues is largely due to the presence of feedback terms and denominator functions that correspond to the linear stabilizing feedback terms of the continuous-time networks. With a similar architectural design, the method has been applied further by $\mathrm{Akça}$ et al. [1, $\mathrm{Covachev}$ et al. [7,

2000 Mathematics Subject Classification: 39A11, 65Q05, 92B20.

Keywords: Cohen-Grossberg neural networks, delays, discrete-time analogues, Lyapunov exponents. 
Gopalsamy [1] and Moham a d22] for simulating the dynamical characteristics of continuous-time neural networks that are subject to impulsive state displacements at fixed moments of time.

C o h e n- Gross b e r g neural network [6] and its various generalizations with or without transmission delays and impulsive state displacements have been the subject of intense investigation recently [2, 4, 5, 27, 31, 32. In a Cohen-Grossberg neural network model, the feedback terms consist of amplification and stabilizing functions which are generally nonlinear. These terms provide the model with a special kind of generalization wherein many neural network models that are capable for content addressable memory such as additive neural networks, cellular neural networks and bidirectional associative memory networks and also biological models such as Lotka-Volterra models of population dynamics are included as special cases.

Despite the generality, the nonlinear feedback terms of the networks are not cohesive with a direct extension of the existing semi-discretization method for obtaining the corresponding discrete-time analogues. In pursuance of the extension, a systematic approach of the semi-discretization method is given in this paper for obtaining the corresponding analogue of a Cohen-Grossberg neural network with distributed transmission delays and impulsive state displacements at fixed moments of time. The dynamical characteristics of the analogue in the general norm $\|\cdot\|_{p}(p \geq 1$ is a real number) are subsequently investigated by exploiting an appropriate Lyapunov sequence and properties of a monotone matrix (i.e., $M$-matrix), and this leads to obtaining a Lyapunov exponent expressed in the form of a relation involving the magnitude and frequency of the impulses. A corollary is given to demonstrate the effectiveness of the main result.

\section{Impulsive continuous-time Cohen-Grossberg neural network}

The impulsive continuous time neural network consists of $m$ elementary processing units (or neurons) whose state variables $x_{i}(i=\overline{1, m})$ are governed by

$$
\begin{aligned}
& \frac{\mathrm{d} x_{i}(t)}{\mathrm{d} t}=a_{i}\left(x_{i}(t)\right)\left[-b_{i}\left(x_{i}(t)\right)+\sum_{j=1}^{m} c_{i j} f_{j}\left(x_{j}\left(t-\tau_{i j}\right)\right)\right. \\
& \left.\quad+\sum_{j=1}^{m} d_{i j} \int_{0}^{\infty} K_{i j}(s) g_{j}\left(x_{j}(t-s)\right) \mathrm{d} s+I_{i}\right], \quad t>t_{0}, t \neq t_{k}, \\
& \Delta x_{i}\left(t_{k}\right)=r_{i k}\left(x_{i}\left(t_{k}^{-}\right)\right), \quad i=\overline{1, m}, \quad k \in \mathbb{N}=\{1,2,3, \ldots\}
\end{aligned}
$$




\section{DISCRETE COHEN-GROSSBERG NEURAL NETWORKS}

with initial values prescribed by piecewise-continuous functions $x_{i}(s)=\phi_{i}(s)$ which are bounded for $s \in\left(-\infty, t_{0}\right]$. In (1) $a_{i}\left(x_{i}\right)$ denotes an amplification function; $b_{i}\left(x_{i}\right)$ denotes an appropriate function which supports the stabilizing (or negative) feedback term $-a_{i}\left(x_{i}\right) b_{i}\left(x_{i}\right)$ of the unit $i ; f_{j}\left(x_{j}\right), g_{j}\left(x_{j}\right)$ denote activation functions; the parameters $c_{i j}, d_{i j}$ are real numbers that represent the weights (or strengths) of the synaptic connections between the $j$ th unit and the $i$ th unit; the real constant $I_{i}$ represents an input signal introduced from outside the network to the $i$ th unit; $\tau_{i j}$ are nonnegative real numbers whose presence indicates the delayed transmission of signals at time $t-\tau_{i j}$ from the $j$ th unit to the unit $i$; and the delay kernels $K_{i j}$ incorporate the fading past effects (or fading memories) of the $j$ th unit on the $i$ th unit; $\Delta x_{i}\left(t_{k}\right)=x_{i}\left(t_{k}^{+}\right)-x_{i}\left(t_{k}^{-}\right)$ denote impulsive state displacements characterized by the nonlinear functions $r_{i k}\left(x_{i}\left(t_{k}^{-}\right)\right)$at fixed moments of time $t_{k}, k \in \mathbb{N}$. Here it is assumed that $x_{i}\left(t_{k}^{+}\right)=$ $\lim _{t \rightarrow t_{k}^{+}} x_{i}(t)$ and $x_{i}\left(t_{k}^{-}\right)=\lim _{t \rightarrow t_{k}^{-}} x_{i}(t)$, and the sequence of times $\left\{t_{k}\right\}_{k=1}^{\infty}$ satisfies $t_{0}<t_{1}<t_{2}<\cdots<t_{k} \rightarrow \infty$ as $k \rightarrow \infty$ and $\Delta t_{k}=t_{k}-t_{k-1} \geq \theta$, where $\theta>0$ denotes the minimum time interval between successive impulses. In other words, the value $\theta>0$ means that the impulses do not occur too often, but $\theta=\infty$ means that the network (11) is free of impulses.

The assumptions that accompany the impulsive network (11) are given as follows:

$\mathbf{A}_{1}$ : The amplification functions $a_{i}: \mathbb{R} \rightarrow \mathbb{R}^{+}$are continuous and bounded in the sense that

$$
0<\underline{a}_{i} \leq a_{i}(u) \leq \bar{a}_{i} \quad \text { for } \quad u \in \mathbb{R}, \quad i=\overline{1, m} .
$$

$\mathbf{A}_{2}$ : The stabilizing functions $b_{i}: \mathbb{R} \rightarrow \mathbb{R}$ are continuous and monotone increasing, namely,

$$
0<\underline{b}_{i} \leq \frac{b_{i}(u)-b_{i}(v)}{u-v} \quad \text { for } \quad u \neq v, \quad u, v \in \mathbb{R}, \quad i=\overline{1, m} .
$$

$\mathbf{A}_{3}$ : The activation functions $f_{j}, g_{j}: \mathbb{R} \rightarrow \mathbb{R}$ with $f_{j}(0)=g_{j}(0)=0$ are Lipschitz continuous in the sense of

$L_{j}^{f}=\sup _{u \neq v}\left|\frac{f_{j}(u)-f_{j}(v)}{u-v}\right|, L_{j}^{g}=\sup _{u \neq v}\left|\frac{g_{j}(u)-g_{j}(v)}{u-v}\right| \quad$ for $\quad u, v \in \mathbb{R}, \quad i=\overline{1, m}$, where $L_{j}^{f}, L_{j}^{g}$ denote positive constants.

$\mathbf{A}_{4}$ : For the impulse functions $r_{i k}: \mathbb{R} \rightarrow \mathbb{R}$ there exist positive numbers $\gamma_{i k}$ such that

$$
\gamma_{i k}=\sup _{u \neq v}\left|\frac{r_{i k}(u)-r_{i k}(v)}{u-v}\right| \quad \text { for } \quad u, v \in \mathbb{R}, \quad k \in \mathbb{N}, \quad i=\overline{1, m} .
$$




\section{SANNAY MOHAMAD — HAYDAR AKÇA — VALÉRY COVACHEV}

$\mathbf{A}_{5}$ : The delay kernels $K_{i j}:[0, \infty) \rightarrow[0, \infty)$ are piecewise continuous functions that satisfy

$$
\int_{0}^{\infty} K_{i j}(s) \mathrm{d} s=\kappa_{i j} \text { and } \int_{0}^{\infty} K_{i j}(s) e^{\nu_{0} s} \mathrm{~d} s<\infty,
$$

where $\kappa_{i j}$ denote nonnegative constants and $\nu_{0}$ is some positive number.

Under these assumptions and the given initial conditions, there is a unique solution of the impulsive network (11). The solution is a vector

$$
x(t)=\left(x_{1}(t), x_{2}(t), \ldots, x_{m}(t)\right)^{T}
$$

in which $x_{i}(t)$ are piecewise continuous for $t \in\left(t_{0}, \beta\right)$, where $\beta>t_{0}$ is some positive number, possibly $\infty$, such that the limits $x_{i}\left(t_{k}^{+}\right)$and $x_{i}\left(t_{k}^{-}\right)$exist and $x_{i}(t)$ are differentiable for $t \in\left(t_{k-1}, t_{k}\right) \subset\left(t_{0}, \beta\right)$. An equilibrium point of the impulsive network (11) is denoted by $x^{*}=\left(x_{1}^{*}, x_{2}^{*}, \ldots, x_{m}^{*}\right)^{T}$ whereby the components $x_{i}^{*}$ are governed by the algebraic system

$$
b_{i}\left(x_{i}^{*}\right)=\sum_{j=1}^{m} c_{i j} f_{j}\left(x_{j}^{*}\right)+\sum_{j=1}^{m} d_{i j} \kappa_{i j} g_{j}\left(x_{j}^{*}\right)+I_{i}, \quad i=\overline{1, m},
$$

and satisfy the equalities

$$
r_{i k}\left(x_{i}^{*}\right)=0, \quad k \in \mathbb{N}, \quad i=\overline{1, m} .
$$

\section{Formulation of an impulsive discrete-time analogue}

For a non-impulsive neural network model with linear stabilizing feedback terms given by $-\hat{b}_{i} x_{i}(t)$, that is, the terms are obtained from (1) by letting $a_{i}\left(x_{i}(t)\right)=1$ and $b_{i}\left(x_{i}(t)\right)=\hat{b}_{i} x_{i}(t)$, where $\hat{b}_{i}$ are positive constants, the application of the existing semi-discretization method 25] yields a discrete-time analogue with corresponding feedback terms given by $e^{-\hat{b}_{i} h} x_{i}(n)$, where $h$ denotes a fixed time-step of the discretization. These terms together with the associated denominator functions given by $\hat{\psi}_{i}(h)=\frac{1-e^{-\hat{b}_{i} h}}{\hat{b}_{i}}$ provide the analogue with features that are conducive for preserving the dynamical characteristics of the continuous-time model, without requiring to restrict the magnitude of the timestep and introduce additional restrictions not envisaged in the continuous-time models.

Till now, the semi-discretization model has not been exploited for obtaining a discrete-time analogue of Cohen-Grossberg neural network mainly due to the nonlinearity of the feedback terms $-a_{i}\left(x_{i}\right) b_{i}\left(x_{i}\right)$. An appropriate extension of the 


\section{DISCRETE COHEN-GROSSBERG NEURAL NETWORKS}

method is presented here. We begin by rewriting the differential system in (11) as

$$
\begin{aligned}
\frac{\mathrm{d} x_{i}(t)}{\mathrm{d} t}= & -\beta_{i} x_{i}(t)+\beta_{i} x_{i}(t)+a_{i}\left(x_{i}(t)\right)\left[-b_{i}\left(x_{i}(t)\right)+\sum_{j=1}^{m} c_{i j} f_{j}\left(x_{j}\left(t-\tau_{i j}\right)\right)\right. \\
& \left.+\sum_{j=1}^{m} d_{i j} \int_{0}^{\infty} K_{i j}(s) g_{j}\left(x_{j}(t-s)\right) \mathrm{d} s+I_{i}\right], \quad i=\overline{1, m}, t>t_{0}, t \neq t_{k},
\end{aligned}
$$

where $\beta_{i}=\underline{a}_{i} \underline{b}_{i}>0$. Let the value $h \in(0, \theta)$ be fixed, and $n_{0}=\left[\frac{t_{0}}{h}\right], n=\left[\frac{t}{h}\right]$, $\sigma_{i j}=\left[\frac{\tau_{i j}}{h}\right]$ and $\ell=\left[\frac{s}{h}\right]$, where $[r]$ denotes the greatest integer contained in the real number $r$. On any interval $[n h,(n+1) h)$ not containing a moment of impulse effect $t_{k}$ the equation (41) can be approximated by equations with constant arguments of the form

$$
\begin{aligned}
& \frac{\mathrm{d} x_{i}(t)}{\mathrm{d} t}=-\beta_{i} x_{i}(t)+\beta_{i} x_{i}\left(\left[\frac{t}{h}\right] h\right) \\
& +a_{i}\left(x_{i}\left(\left[\frac{t}{h}\right] h\right)\right)\left[-b_{i}\left(x_{i}\left(\left[\frac{t}{h}\right] h\right)\right)+\sum_{j=1}^{m} c_{i j} f_{j}\left(x_{j}\left(\left[\frac{t}{h}\right] h-\left[\frac{\tau_{i j}}{h}\right] h\right)\right)\right. \\
& \left.+\sum_{j=1}^{m} d_{i j} \sum_{\left[\frac{s}{h}\right]=1}^{\infty} \mathcal{K}_{i j}\left(\left[\frac{s}{h}\right] h\right) g_{j}\left(x_{j}\left(\left[\frac{t}{h}\right] h-\left[\frac{s}{h}\right] h\right)\right)+I_{i}\right], \quad i=\overline{1, m},
\end{aligned}
$$

with

$$
\begin{gathered}
{\left[\frac{t}{h}\right] h=n h \rightarrow t, \quad\left[\frac{\tau_{i j}}{h}\right] h=\sigma_{i j} h \rightarrow \tau_{i j}, \quad\left[\frac{s}{h}\right] h=\ell h \rightarrow s,} \\
\sum_{\left[\frac{s}{h}\right]=1}^{\infty} \mathcal{K}_{i j}\left(\left[\frac{s}{h}\right] h\right) g_{j}\left(x_{j}\left(\left[\frac{t}{h}\right] h-\left[\frac{s}{h}\right] h\right)\right) \longrightarrow \int_{0}^{\infty} K_{i j}(s) g_{j}\left(x_{j}(t-s)\right) \mathrm{d} s
\end{gathered}
$$

for a fixed time $t$ as $h \rightarrow 0$. Moreover,

$\mathbf{A}_{5}^{\prime}$ : The delay kernels $\mathcal{K}_{i j}: \mathbb{N} \rightarrow[0, \infty)(i, j=\overline{1, m})$ satisfy

$$
\sum_{\ell=1}^{\infty} \mathcal{K}_{i j}(\ell)=\kappa_{i j} \quad \text { and } \quad \sum_{\ell=1}^{\infty} \mathcal{K}_{i j}(\ell) e^{\nu \ell h}<\infty,
$$

where the positive real number $\nu=\nu(h)$ for a given $h \in(0, \theta)$ is related to the positive number $\nu_{0}$ (cf. assumption $\mathbf{A}_{5}$ ) by $\nu \rightarrow \nu_{0}$ (from below) as $h \rightarrow 0$. 


\section{SANNAY MOHAMAD — HAYDAR AKÇA — VALÉRY COVACHEV}

For simplicity, we write system (15) as

$$
\begin{aligned}
\frac{\mathrm{d} x_{i}(t)}{\mathrm{d} t}= & -\beta_{i} x_{i}(t)+\beta_{i} x_{i}(n) \\
& +a_{i}\left(x_{i}(n)\right)\left[-b_{i}\left(x_{i}(n)\right) \sum_{j=1}^{m}+\sum_{j=1}^{m} c_{i j} f_{j}\left(x_{j}\left(n-\sigma_{i j}\right)\right)\right. \\
& \left.+\sum_{j=1}^{m} d_{i j} \sum_{\ell=1}^{\infty} \mathcal{K}_{i j}(\ell) g_{j}\left(x_{j}(n-\ell)\right)+I_{i}\right]
\end{aligned}
$$

for $i=\overline{1, m}, t \in[n h,(n+1) h), t \neq t_{k}, n=n_{0}, n_{0}+1, \ldots$, wherein the notation $w(n) \equiv w(n h)$ has been adopted. Upon integrating (6) over the interval $[n h,(n+1) h)$, one obtains a discrete analogue of the differential system in (1) given by

$$
\begin{aligned}
x_{i}(n+1)= & e^{-\beta_{i} h} x_{i}(n)+\psi_{i}(h)\left\{\beta_{i} x_{i}(n)+a_{i}\left(x_{i}(n)\right)\left[-b_{i}\left(x_{i}(n)\right)\right.\right. \\
& \left.\left.+\sum_{j=1}^{m} c_{i j} f_{j}\left(x_{j}\left(n-\sigma_{i j}\right)\right)+\sum_{j=1}^{m} d_{i j} \sum_{\ell=1}^{\infty} \mathcal{K}_{i j}(\ell) g_{j}\left(x_{j}(n-\ell)\right)+I_{i}\right]\right\}
\end{aligned}
$$

for $i=\overline{1, m}, n \geq n_{0}, n \neq n_{k}$, where $\psi_{i}(h)=\frac{1-e^{-\beta_{i} h}}{\beta_{i}}$ denotes the associated denominator function. Observe that $0<\psi_{i}(h)<\frac{1}{\beta_{i}}$ for $0<h<\theta$ and $\psi_{i}(h) \approx$ $h+O\left(h^{2}\right)$ for small $h>0$.

The analogue (7) is supplemented with an initial vector sequence $\phi(\ell)=$ $\left(\phi_{1}(\ell), \phi_{2}(\ell), \ldots, \phi_{m}(\ell)\right)^{T}$ for $\ell=n_{0}, n_{0}-1, n_{0}-2, \ldots$ and is subject to impulsive state displacements characterized by the map

$$
x_{i}\left(n_{k}^{+}\right)=x_{i}\left(n_{k}^{-}\right)+r_{i k}\left(x_{i}\left(n_{k}^{-}\right)\right), \quad i=\overline{1, m}, \quad k \in \mathbb{N} .
$$

The iterations involved in (17) and (8) are described as follows: The notations $n_{k}^{+}$and $n_{k}^{-}$denote the same integer $n_{k}$ for which the values $x_{i}\left(n_{k}^{-}\right)$generated by system (77) at time $n=n_{k}-1$ are mapped impulsively by (8) to give the values $x_{i}\left(n_{k}^{+}\right)$. The mapped values $x_{i}\left(n_{k}^{+}\right)$together with the past values $x_{i}(\ell)$ for $\ell=$ $n_{k}-1, n_{k}-2, \ldots$ are then supplied back to system (7) as initial values required for the next successive iterations of $x_{i}(n+1)$ for $n=n_{k}, n_{k}+1, \ldots, n_{k+1}-1$. The existence of a unique solution $x(n)=\left(x_{1}(n), x_{2}(n), \ldots, x_{m}(n)\right)^{T}$ of the impulsive analogue (7), (8) for $n>n_{0}$ is therefore justified.

The impulsive map (8) was introduced in 22, wherein the impulse functions $r_{i k}$ were defined linearly by $r_{i k}\left(x_{i}\left(n_{k}^{-}\right)\right)=-\delta_{i k}\left(x_{i}\left(n_{k}^{-}\right)-x_{i}^{*}\right)$ for $i=\overline{1, m}, k \in \mathbb{N}$ with $\delta_{i k}$ denoting real numbers. An iteration scheme similar to (77), (8) was 


\section{DISCRETE COHEN-GROSSBERG NEURAL NETWORKS}

introduced in [8]. This map, for a given $h \in(0, \theta)$, differs from the impulsive state displacements described by difference equations of the form

$$
x_{i}\left(n_{k}+1\right)=x_{i}\left(n_{k}\right)+r_{i k}\left(x_{i}\left(n_{k}\right)\right), \quad i=\overline{1, m}, \quad k \in \mathbb{N},
$$

considered in [1, 7, 11. However, in the limit $h \rightarrow 0$, both characterizations operate in a similar manner in accordance with the continuous-time characterization (11) at the impulse moment $t=t_{k}$.

\section{Exponential stability of equilibria}

One can verify that an equilibrium point $x^{*}=\left(x_{1}^{*}, x_{2}^{*}, \ldots, x_{m}^{*}\right)^{T}$ of the impulsive analogue (77), (8) satisfies the same algebraic system (2) and (3) under the assumptions $\mathbf{A}_{1}-\mathbf{A}_{4}, \mathbf{A}_{5}^{\prime}$ for any given value $h \in(0, \theta)$. To prove the global exponential stability of the point $x^{*}$ we will need the following definition and lemmas.

Definition 1 ([33]). A real matrix $A=\left(a_{i j}\right)_{m \times m}$ is said to be an $M$-matrix if $a_{i j} \leq 0$ for $i, j=\overline{1, m}, i \neq j$ and all successive principle minors of $A$ are positive.

Lemma $1([3])$. Let $A=\left(a_{i j}\right)_{m \times m}$ be a real matrix with non-positive off-diagonal elements. Then $A$ is an $M$-matrix if and only if one of the following conditions holds:

(1) There exists a vector $\xi=\left(\xi_{1}, \xi_{2}, \ldots, \xi_{m}\right)^{T}$ with $\xi_{i}>0$ such that every component of $\xi^{T} A$ is positive-that is, $\sum_{i=1}^{m} \xi_{i} a_{i j}>0, j=\overline{1, m}$.

(2) There exists a vector $\xi=\left(\xi_{1}, \xi_{2}, \ldots, \xi_{m}\right)^{T}$ with $\xi_{i}>0$ such that every component of $A \xi$ is positive-that is, $\sum_{j=1}^{m} a_{i j} \xi_{j}>0, i=\overline{1, m}$.

For more details about $M$-matrices the reader is referred to [9, 13.

Lemma 2 ([10]). A locally invertible $C^{0}$ map $H: \mathbb{R}^{m} \rightarrow \mathbb{R}^{m}$ is a homeomorphism of $\mathbb{R}^{m}$ onto itself if and only if it is proper.

In fact, this assertion is due to $\mathrm{H}$ a d a m a r d [12]. A mapping is proper if the pre-image of every compact is compact. In the finite-dimensional case it suffices to show that $\|H(x)\| \rightarrow \infty$ as $\|x\| \rightarrow \infty$.

Our first task is to prove the existence and uniqueness of the solution $x^{*}$ of the algebraic system (2).

Theorem 1. Let $p \geq 1$ be a real number, the value $h \in(0, \theta)$ be fixed and the assumptions $\mathbf{A}_{1}-\mathbf{A}_{4}, \mathbf{A}_{5}^{\prime}$ hold. Suppose the matrix

$$
\Xi_{0}=B_{0}-\frac{p-1}{p}\left(C_{0}^{*}+D_{0}^{*}\right)-\frac{1}{p}\left(C_{0} L^{f}+D_{0} L^{g}\right)
$$


is an $M$-matrix, where

$$
\begin{aligned}
B_{0} & =\operatorname{diag}\left(\underline{b}_{1}, \underline{b}_{2}, \ldots, \underline{b}_{m}\right), \quad C_{0}=\left(\left|c_{i j}\right|\right)_{m \times m}, \quad D_{0}=\left(\left|d_{i j}\right| \kappa_{i j}\right)_{m \times m}, \\
C_{0}^{*} & =\operatorname{diag}\left(\sum_{j=1}^{m}\left|c_{1 j}\right| L_{j}^{f}, \sum_{j=1}^{m}\left|c_{2 j}\right| L_{j}^{f}, \ldots, \sum_{j=1}^{m}\left|c_{m j}\right| L_{j}^{f}\right), \\
D_{0}^{*} & =\operatorname{diag}\left(\sum_{j=1}^{m}\left|d_{1 j}\right| \kappa_{1 j} L_{j}^{g}, \sum_{j=1}^{m}\left|d_{2 j}\right| \kappa_{2 j} L_{j}^{g}, \ldots, \sum_{j=1}^{m}\left|d_{m j}\right| \kappa_{m j} L_{j}^{g}\right), \\
L^{f} & =\operatorname{diag}\left(L_{1}^{f}, L_{2}^{f}, \ldots, L_{m}^{f}\right), \quad L^{g}=\operatorname{diag}\left(L_{1}^{g}, L_{2}^{g}, \ldots, L_{m}^{g}\right) .
\end{aligned}
$$

Then the algebraic system (2) has a unique solution $x^{*}=\left(x_{1}^{*}, x_{2}^{*}, \ldots, x_{m}^{*}\right)^{T}$.

Id ea of the proof. Define a mapping $F: \mathbb{R}^{m} \rightarrow \mathbb{R}^{m}$ by

$$
F(x)=\left(F_{1}(x), F_{2}(x), \ldots, F_{m}(x)\right)^{T} \quad \text { for } \quad x \in \mathbb{R}^{m},
$$

where

$$
F_{i}(x)=-b_{i}\left(x_{i}\right)+\sum_{j=1}^{m} c_{i j} f_{j}\left(x_{j}\right)+\sum_{j=1}^{m} d_{i j} \kappa_{i j} g_{j}\left(x_{j}\right)+I_{i}, \quad i=\overline{1, m} .
$$

The space $\mathbb{R}^{m}$ is endowed with the norm $\|x\|_{p}=\left(\sum_{i=1}^{m}\left|x_{i}\right|^{p}\right)^{1 / p}$. Under the assumptions $\mathbf{A}_{2}, \mathbf{A}_{3}, F(x) \in C^{0}$. It is known that if $F(x) \in C^{0}$ is a homeomorphism of $\mathbb{R}^{m}$, then there is a unique point $x^{*}=\left(x_{1}^{*}, x_{2}^{*}, \ldots, x_{m}^{*}\right)^{T} \in \mathbb{R}^{m}$ such that $F\left(x^{*}\right)=0$, that is, $F_{i}\left(x^{*}\right)=0, i=\overline{1, m}$.

To demonstrate the one-to-one property of $F$, we take arbitrary vectors $x, y \in$ $\mathbb{R}^{m}$ and assume that $F(x)=F(y)$. From

$$
\begin{aligned}
& b_{i}\left(x_{i}\right)-b_{i}\left(y_{i}\right)=\sum_{j=1}^{m} c_{i j}\left(f_{j}\left(x_{j}\right)-f_{j}\left(y_{j}\right)\right) \\
& +\sum_{j=1}^{m} d_{i j} \kappa_{i j}\left(g_{j}\left(x_{j}\right)-g_{j}\left(y_{j}\right)\right), \quad i=\overline{1, m},
\end{aligned}
$$

one obtains

$$
\underline{b}_{i}\left|x_{i}-y_{i}\right| \leq \sum_{j=1}^{m}\left|c_{i j}\right| L_{j}^{f}\left|x_{j}-y_{j}\right|+\sum_{j=1}^{m}\left|d_{i j}\right| \kappa_{i j} L_{j}^{g}\left|x_{j}-y_{j}\right|, \quad i=\overline{1, m},
$$

under the given assumptions. Multiplying both sides of the last inequality by $\left|x_{i}-y_{i}\right|^{p-1}$ and applying the inequality

$$
\eta_{1}^{p-1} \eta_{2} \leq \frac{p-1}{p} \eta_{1}^{p}+\frac{1}{p} \eta_{2}^{p}, \quad \eta_{1}, \eta_{2} \geq 0, \quad p \geq 1
$$




\section{DISCRETE COHEN-GROSSBERG NEURAL NETWORKS}

we derive

$$
\begin{aligned}
\underline{b}_{i}\left|x_{i}-y_{i}\right|^{p} \leq & \sum_{j=1}^{m}\left|c_{i j}\right| L_{j}^{f}\left|x_{i}-y_{i}\right|^{p-1}\left|x_{j}-y_{j}\right| \\
& +\sum_{j=1}^{m}\left|d_{i j}\right| \kappa_{i j} L_{j}^{g}\left|x_{i}-y_{i}\right|^{p-1}\left|x_{j}-y_{j}\right| \\
\leq & \sum_{j=1}^{m}\left|c_{i j}\right| L_{j}^{f}\left(\frac{p-1}{p}\left|x_{i}-y_{i}\right|^{p}+\frac{1}{p}\left|x_{j}-y_{j}\right|^{p}\right) \\
& +\sum_{j=1}^{m}\left|d_{i j}\right| \kappa_{i j} L_{j}^{g}\left(\frac{p-1}{p}\left|x_{i}-y_{i}\right|^{p}+\frac{1}{p}\left|x_{j}-y_{j}\right|^{p}\right), \quad i=\overline{1, m},
\end{aligned}
$$

which can be expressed as

$$
\Xi_{0}\left(\left|x_{1}-y_{1}\right|^{p},\left|x_{2}-y_{2}\right|^{p}, \ldots,\left|x_{m}-y_{m}\right|^{p}\right)^{T} \leq 0 .
$$

The assertion $x_{i}=y_{i}, i=\overline{1, m}$, follows by virtue of $\Xi_{0}$ being an $M$-matrix. Thus $F(x)=F(y)$ implies $x=y$.

Finally, we show that $\|F(x)\|_{p} \rightarrow \infty$ as $\|x\|_{p} \rightarrow \infty$. According to Lemma 2 $F(x) \in C^{0}$ is a homeomorphism of $\mathbb{R}^{m}$. Thus there is a unique point $x^{*} \in \mathbb{R}^{m}$ such that $F\left(x^{*}\right)=0$. The point represents a unique solution of the algebraic system (2).

The next task is to investigate the global exponential stability characteristics of the unique equilibrium point $x^{*}$ of the impulse analogue (77), (8) for a fixed time-step $h \in(0, \theta)$. Upon introducing the translations

$$
\begin{gathered}
u_{i}(n)=x_{i}(n)-x_{i}^{*}, \quad \varphi_{i}(\ell)=\phi_{i}(\ell)-x_{i}^{*}, \quad \tilde{a}_{i}\left(u_{i}(n)\right)=a_{i}\left(u_{i}(n)+x_{i}^{*}\right), \\
\tilde{b}_{i}\left(u_{i}(n)\right)=b_{i}\left(u_{i}(n)+x_{i}^{*}\right)-b_{i}\left(x_{i}^{*}\right), \quad \tilde{f}_{j}\left(u_{j}(n)\right)=f_{j}\left(u_{j}(n)+x_{j}^{*}\right)-f_{j}\left(x_{j}^{*}\right), \\
\tilde{g}_{j}\left(u_{j}(n)\right)=g_{j}\left(u_{j}(n)+x_{j}^{*}\right)-g_{j}\left(x_{j}^{*}\right), \quad \tilde{r}_{i k}\left(u_{i}\left(n_{k}^{-}\right)\right)=r_{i k}\left(u_{i}\left(n_{k}^{-}\right)+x_{i}^{*}\right)+u_{i}\left(n_{k}^{-}\right),
\end{gathered}
$$

one obtains

$$
\begin{aligned}
u_{i}(n+1)= & e^{-\beta_{i} h} u_{i}(n) \\
& +\psi_{i}(h)\left\{\beta_{i} u_{i}(n)+\tilde{a}_{i}\left(u_{i}(n)\right)\left[-\tilde{b}_{i}\left(x_{i}(n)\right)+\sum_{j=1}^{m} c_{i j} \tilde{f}_{j}\left(u_{j}\left(n-\sigma_{i j}\right)\right)\right.\right. \\
& \left.\left.+\sum_{j=1}^{m} d_{i j} \sum_{\ell=1}^{\infty} \mathcal{K}_{i j}(\ell) \tilde{g}_{j}\left(x_{j}(n-\ell)\right)\right]\right\}, \quad i=\overline{1, m}, \quad n \geq n_{0}, \quad n \neq n_{k}, \\
u_{i}\left(n_{k}^{+}\right)= & \tilde{r}_{i k}\left(u_{i}\left(n_{k}^{-}\right)\right), \quad i=\overline{1, m}, \quad k \in \mathbb{N} .
\end{aligned}
$$




\section{SANNAY MOHAMAD — HAYDAR AKÇA — VALÉRY COVACHEV}

This system inherits the assumptions $\mathbf{A}_{\mathbf{1}}-\mathbf{A}_{\mathbf{4}}, \mathbf{A}_{\mathbf{5}}^{\prime}$ given before. In particular,

$$
\tilde{r}_{i k}(0)=0 \quad \text { for } \quad i=\overline{1, m}, \quad k \in \mathbb{N}
$$

and

$$
\left|\tilde{r}_{i k}(u)\right| \leq\left(1+\gamma_{i k}\right)|u| \quad \text { for } \quad i=\overline{1, m}, \quad k \in \mathbb{N}, \quad u \in \mathbb{R},
$$

where $\gamma_{i k}$ denote positive real numbers.

Due to the equivalence between the systems (10) and (7), (8), it suffices to examine the exponential stability characteristics of the trivial equilibrium point $u^{*}=0$ of the impulsive analogue (10). The main result is given by the following theorem.

TheOREM 2. Let $p \geq 1$ be a real number, the value $h \in(0, \theta)$ be fixed and the assumptions $\mathbf{A}_{1}-\mathbf{A}_{4}, \mathbf{A}_{5}^{\prime}$ hold. Suppose the matrix

$$
\Xi_{1}=B_{1}-\frac{p-1}{p}\left(C_{1}^{*}+D_{1}^{*}\right)-\frac{1}{p}\left(C_{1} L^{f}+D_{1} L^{g}\right)
$$

is an $M$-matrix, where

$$
\begin{aligned}
B_{1} & =\operatorname{diag}\left(\underline{a}_{1} \underline{b}_{1}, \underline{a}_{2} \underline{b}_{2}, \ldots, \underline{a}_{m} \underline{b}_{m}\right), \quad C_{1}=\left(\bar{a}_{i}\left|c_{i j}\right|\right)_{m \times m}, D_{1}=\left(\bar{a}_{i}\left|d_{i j}\right| \kappa_{i j}\right)_{m \times m}, \\
C_{1}^{*} & =\operatorname{diag}\left(\sum_{j=1}^{m} \bar{a}_{1}\left|c_{1 j}\right| L_{j}^{f}, \sum_{j=1}^{m} \bar{a}_{2}\left|c_{2 j}\right| L_{j}^{f}, \ldots, \sum_{j=1}^{m} \bar{a}_{m}\left|c_{m j}\right| L_{j}^{f}\right) \\
D_{1}^{*} & =\operatorname{diag}\left(\sum_{j=1}^{m} \bar{a}_{1}\left|d_{1 j}\right| \kappa_{1 j} L_{j}^{g}, \sum_{j=1}^{m} \bar{a}_{2}\left|d_{2 j}\right| \kappa_{2 j} L_{j}^{g}, \ldots, \sum_{j=1}^{m} \bar{a}_{m}\left|d_{m j}\right| \kappa_{m j} L_{j}^{g}\right) .
\end{aligned}
$$

Suppose, further, that there exist positive numbers $\Lambda>1$ and $\mu$ satisfying $\frac{\ln \Lambda}{\theta}<$ $\mu<\nu$ for which

$$
1+\gamma_{i k} \leq \Lambda \quad \text { for } \quad i=\overline{1, m}, \quad k \in \mathbb{N} .
$$

Then the impulsive analogue (10) is globally exponentially stable with a Lyapunov exponent $\mu-\frac{\ln \Lambda}{\theta}$, namely,

$$
\left[\sum_{i=1}^{m}\left|u_{i}(n)\right|^{p}\right]^{1 / p} \leq \Omega e^{-\left(\mu-\frac{\ln \Lambda}{\theta}\right) h\left(n-n_{0}\right)}\left[\sum_{i=1}^{m} \sup _{\ell \leq n_{0}}\left|\varphi_{i}(\ell)\right|^{p}\right]^{1 / p}
$$

for $n \geq n_{0}$, where $\Omega \geq 1$ denotes a constant.

Sketch of the proof. The property of the $M$-matrix $\Xi_{1}$ lends itself towards ascertaining that $\Xi_{0}$ is an $M$-matrix. Thus the existence and uniqueness of the equilibrium point $x^{*}$ is assured by Theorem 1 . 


\section{DISCRETE COHEN-GROSSBERG NEURAL NETWORKS}

On applying the given assumptions to (10),

$$
\begin{aligned}
\left|u_{i}(n+1)\right| \leq & e^{-\beta_{i} h}\left|u_{i}(n)\right| \\
& +\psi_{i}(h)\left\{\sum_{j=1}^{m} \bar{a}_{i}\left|c_{i j}\right| L_{j}^{f}\left|u_{j}\left(n-n_{i j}\right)\right|\right. \\
& \left.+\sum_{j=1}^{m} \bar{a}_{i}\left|d_{i j}\right| \sum_{\ell=1}^{\infty} \mathcal{K}_{i j}(\ell) L_{j}^{g}\left|u_{j}(n-\ell)\right|\right\}, \quad i=\overline{1, m}, n \geq n_{0}, n \neq n_{k} .
\end{aligned}
$$

Recall that $\beta_{i}=\underline{a}_{i} \underline{b}_{i}>0$ and $\psi_{i}(h)=\frac{1-e^{-\beta_{i} h}}{\beta_{i}}>0$. From the $M$-matrix $\Xi_{1}$ and the property (1) of Lemma 1 one has that there is a positive vector $\xi=\left(\xi_{1}, \xi_{2}, \ldots, \xi_{m}\right)^{T}$ for which

$$
\begin{aligned}
\xi_{i} \underline{a}_{i} \underline{b}_{i} & -\frac{p-1}{p} \sum_{j=1}^{m} \xi_{i} \bar{a}_{i}\left|c_{i j}\right| L_{j}^{f}-\frac{p-1}{p} \sum_{j=1}^{m} \xi_{i} \bar{a}_{i}\left|d_{i j}\right| \kappa_{i j} L_{j}^{g} \\
& -\frac{1}{p} \sum_{j=1}^{m} \xi_{j} \bar{a}_{j}\left|c_{j i}\right| L_{i}^{f}-\frac{1}{p} \sum_{j=1}^{m} \xi_{j} \bar{a}_{j}\left|d_{j i}\right| \kappa_{j i} L_{i}^{g}>0, \quad i=\overline{1, m} .
\end{aligned}
$$

Let us introduce a perturbation $\mu=\mu(h)$ for a fixed value $h \in(0, \theta)$ such that $0<\mu<\min \left\{\min _{i=\overline{1, m}} \beta_{i}, \nu\right\}$ and

$$
\begin{aligned}
\frac{e^{\left(\mu-\beta_{i}\right) h}-1}{\psi_{i}(h)} & +\frac{p-1}{p} \sum_{j=1}^{m} \bar{a}_{i}\left|c_{i j}\right| L_{j}^{f} e^{\mu\left(\sigma_{i j}+1\right) h} \\
& +\frac{p-1}{p} \sum_{j=1}^{m} \bar{a}_{i}\left|d_{i j}\right| L_{j}^{g} \sum_{\ell=1}^{\infty} \mathcal{K}_{i j}(\ell) e^{\mu(\ell+1) h} \\
& +\frac{1}{p} \sum_{j=1}^{m} \frac{\xi_{j}}{\xi_{i}} \bar{a}_{j}\left|c_{j i}\right| L_{i}^{f} e^{\mu\left(\sigma_{j i}+1\right) h} \\
& +\frac{1}{p} \sum_{j=1}^{m} \frac{\xi_{j}}{\xi_{i}} \bar{a}_{j}\left|d_{j i}\right| L_{i}^{g} \sum_{\ell=1}^{\infty} \mathcal{K}_{j i}(\ell) e^{\mu(\ell+1) h} \leq 0, \quad i=\overline{1, m} .
\end{aligned}
$$

Further, let

$$
X_{i}(n)=e^{\mu\left(n-n_{0}\right) h}\left|u_{i}(n)\right| \quad \text { for } \quad i=\overline{1, m}, \quad n \in \mathbb{Z},
$$


into the system (15). We obtain

$$
\begin{aligned}
& X_{i}(n+1) \leq e^{\left(\mu-\beta_{i}\right) h} X_{i}(n) \\
&+\psi_{i}(h)\left\{\sum_{j=1}^{m} \bar{a}_{i}\left|c_{i j}\right| L_{j}^{f} e^{\mu\left(\sigma_{i j}+1\right) h} X_{j}\left(n-\sigma_{i j}\right)\right. \\
&\left.+\sum_{j=1}^{m} \bar{a}_{i}\left|d_{i j}\right| \sum_{\ell=1}^{\infty} \mathcal{K}_{i j}(\ell) L_{j}^{g} e^{\mu(\ell+1) h} X_{j}(n-\ell)\right\}, \\
& \quad i=\frac{1, m}{1, m}, \quad n \geq n_{0}, \quad n \neq n_{k},
\end{aligned}
$$

which can be rearranged as

$$
\begin{aligned}
\frac{\Delta X_{i}(n)}{\psi_{i}(h)} \leq & \frac{e^{\left(\mu-\beta_{i}\right) h}-1}{\psi_{i}(h)} X_{i}(n) \\
& +\sum_{j=1}^{m} \bar{a}_{i}\left|c_{i j}\right| L_{j}^{f} e^{\mu\left(\sigma_{i j}+1\right) h} X_{j}\left(n-\sigma_{i j}\right) \\
& +\sum_{j=1}^{m} \bar{a}_{i}\left|d_{i j}\right| \sum_{\ell=1}^{\infty} \mathcal{K}_{i j}(\ell) L_{j}^{g} e^{\mu(\ell+1) h} X_{j}(n-\ell), \\
& \quad i=\overline{1, m}, \quad n \geq n_{0}, \quad n \neq n_{k} .
\end{aligned}
$$

Define a Lyapunov sequence by

$$
\begin{aligned}
V(n)= & \sum_{i=1}^{m} \xi_{i} \psi_{i}^{-1}(h) X_{i}^{p}(n) \\
& +\sum_{i=1}^{m} \xi_{i} \sum_{j=1}^{m} \bar{a}_{i}\left|c_{i j}\right| L_{j}^{f} e^{\mu\left(\sigma_{i j}+1\right) h} \sum_{\ell=n-\sigma_{i j}}^{n-1} X_{j}^{p}(\ell) \\
& +\sum_{i=1}^{m} \xi_{i} \sum_{j=1}^{m} \bar{a}_{i}\left|d_{i j}\right| L_{j}^{g} \sum_{\ell=1}^{\infty} \mathcal{K}_{i j}(\ell) e^{\mu(\ell+1) h} \sum_{r=n-\ell}^{n-1} X_{j}^{p}(r), \quad n \in \mathbb{Z} .
\end{aligned}
$$

One observes that the value

$$
\begin{aligned}
V\left(n_{0}\right) \leq & \max _{i=\overline{1, m}}\left\{\xi_{i} \psi_{i}^{-1}(h)+\sum_{j=1}^{m} \xi_{j} \bar{a}_{j}\left|c_{j i}\right| L_{i}^{f} e^{\mu\left(\sigma_{j i}+1\right) h} \sigma_{j i}\right. \\
& \left.+\sum_{j=1}^{m} \xi_{j} \bar{a}_{j}\left|d_{j i}\right| L_{i}^{g} \sum_{\ell=1}^{\infty} \mathcal{K}_{j i}(\ell) e^{\mu(\ell+1) h} \ell\right\} \sum_{i=1}^{m} \sup _{r \leq n_{0}} X_{i}^{p}(r)
\end{aligned}
$$

is finite since $\sum_{\ell=1}^{\infty} \mathcal{K}_{j i}(\ell) e^{\mu(\ell+1) h} \ell<\infty$ by virtue of the assumption $\mathbf{A}_{5}^{\prime}$ in which $0<\mu<\nu$ for a fixed $h \in(0, \theta)$ and $\sup _{r \leq n_{0}} X_{i}^{p}(r)<\infty$ due to the boundedness of the initial sequence $\varphi(r)=\phi(r)-x^{*}$ for $r=n_{0}, n_{0}-1, n_{0}-2, \ldots$ 


\section{DISCRETE COHEN-GROSSBERG NEURAL NETWORKS}

Now we estimate the forward difference $\Delta V(n)=V(n+1)-V(n)$ along the solutions of inequality (18), thus

$$
\begin{aligned}
& \Delta V(n)=\sum_{i=1}^{m} \xi_{i} \psi_{i}^{-1}(h) \Delta X_{i}^{p}(n) \\
& +\sum_{i=1}^{m} \xi_{i} \sum_{j=1}^{m} \bar{a}_{i}\left|c_{i j}\right| L_{j}^{f} e^{\mu\left(\sigma_{i j}+1\right) h}\left(X_{j}^{p}(n)-X_{j}^{p}\left(n-\sigma_{i j}\right)\right) \\
& +\sum_{i=1}^{m} \xi_{i} \sum_{j=1}^{m} \bar{a}_{i}\left|d_{i j}\right| L_{j}^{g} \sum_{\ell=1}^{\infty} \mathcal{K}_{i j}(\ell) e^{\mu(\ell+1) h}\left(X_{j}^{p}(n)-X_{j}^{p}(n-\ell)\right) \\
& \leq \sum_{i=1}^{m} \xi_{i} p X_{i}^{p-1}(n)\left\{\frac{e^{\left(\mu-\beta_{i}\right) h}-1}{\psi_{i}(h)} X_{i}(n)+\sum_{j=1}^{m} \bar{a}_{i}\left|c_{i j}\right| L_{j}^{f} e^{\mu\left(\sigma_{i j}+1\right) h} X_{j}\left(n-\sigma_{i j}\right)\right. \\
& \left.+\sum_{j=1}^{m} \bar{a}_{i}\left|d_{i j}\right| L_{j}^{g} \sum_{\ell=1}^{\infty} \mathcal{K}_{i j}(\ell) e^{\mu(\ell+1) h} X_{j}(n-\ell)\right\} \\
& +\sum_{i=1}^{m} \xi_{i} \sum_{j=1}^{m} \bar{a}_{i}\left|c_{i j}\right| L_{j}^{f} e^{\mu\left(\sigma_{i j}+1\right) h}\left(X_{j}^{p}(n)-X_{j}^{p}\left(n-\sigma_{i j}\right)\right) \\
& +\sum_{i=1}^{m} \xi_{i} \sum_{j=1}^{m} \bar{a}_{i}\left|d_{i j}\right| L_{j}^{g} \sum_{\ell=1}^{\infty} \mathcal{K}_{i j}(\ell) e^{\mu(\ell+1) h}\left(X_{j}^{p}(n)-X_{j}^{p}(n-\ell)\right) \\
& \leq \sum_{i=1}^{m} \xi_{i} p\left\{\frac{e^{\left(\mu-\beta_{i}\right) h}-1}{\psi_{i}(h)} X_{i}^{p}(n)\right. \\
& +\sum_{j=1}^{m} \bar{a}_{i}\left|c_{i j}\right| L_{j}^{f} e^{\mu\left(\sigma_{i j}+1\right) h}\left(\frac{p-1}{p} X_{i}^{p}(n)+\frac{1}{p} X_{j}^{p}\left(n-\sigma_{i j}\right)\right) \\
& +\sum_{j=1}^{m} \bar{a}_{i}\left|d_{i j}\right| L_{j}^{g} \sum_{\ell=1}^{\infty} \mathcal{K}_{i j}(\ell) e^{\mu(\ell+1) h}\left(\frac{p-1}{p} X_{i}^{p}(n)+\frac{1}{p} X_{j}^{p}(n-\ell)\right) \\
& +\sum_{j=1}^{m} \bar{a}_{i}\left|c_{i j}\right| L_{j}^{f} e^{\mu\left(\sigma_{i j}+1\right) h}\left(\frac{1}{p} X_{j}^{p}(n)-\frac{1}{p} X_{j}^{p}\left(n-\sigma_{i j}\right)\right) \\
& \left.+\sum_{j=1}^{m} \bar{a}_{i}\left|d_{i j}\right| L_{j}^{g} \sum_{\ell=1}^{\infty} \mathcal{K}_{i j}(\ell) e^{\mu(\ell+1) h}\left(\frac{1}{p} X_{j}^{p}(n)-\frac{1}{p} X_{j}^{p}(n-\ell)\right)\right\} \\
& =\sum_{i=1}^{m} \xi_{i} p\left\{\frac{e^{\left(\mu-\beta_{i}\right) h}-1}{\psi_{i}(h)}+\frac{p-1}{p} \sum_{j=1}^{m} \bar{a}_{i}\left|c_{i j}\right| L_{j}^{f} e^{\mu\left(\sigma_{i j}+1\right) h}\right. \\
& +\frac{p-1}{p} \sum_{j=1}^{m} \bar{a}_{i}\left|d_{i j}\right| L_{j}^{g} \sum_{\ell=1}^{\infty} \mathcal{K}_{i j}(\ell) e^{\mu(\ell+1) h}+\frac{1}{p} \sum_{j=1}^{m} \frac{\xi_{j}}{\xi_{i}} \bar{a}_{j}\left|c_{j i}\right| L_{i}^{f} e^{\mu\left(\sigma_{j i}+1\right) h} \\
& \left.+\frac{1}{p} \sum_{j=1}^{m} \frac{\xi_{j}}{\xi_{i}} \bar{a}_{j}\left|d_{j i}\right| L_{i}^{g} \sum_{\ell=1}^{\infty} \mathcal{K}_{j i}(\ell) e^{\mu(\ell+1) h}\right\} X_{i}^{p}(n)
\end{aligned}
$$


for $n \geq n_{0}, n \neq n_{k}$. By virtue of the condition (16) we find that $\Delta V(n) \leq 0$ for $n \geq n_{0}, n \neq n_{k}$. This means that

$$
V(n) \leq V\left(n_{k-1}^{+}\right) \quad \text { for } \quad n_{k-1} \leq n<n_{k}, \quad k \in \mathbb{N} .
$$

Next,

$$
X_{i}^{p}\left(n_{k-1}^{+}\right) \leq\left(1+\gamma_{i(k-1)}\right)^{p} X_{i}^{p}\left(n_{k-1}^{-}\right) \leq \Lambda^{p} X_{i}^{p}\left(n_{k-1}^{-}\right)
$$

for $k=1,2,3, \ldots$, which implies

$$
V\left(n_{k-1}^{+}\right) \leq \Lambda^{p} V\left(n_{k-1}^{-}\right) \quad \text { for } \quad k \in \mathbb{N} .
$$

From the statements (20) and (21) it follows that

$$
V(n) \leq \Lambda^{(k-1) p} V\left(n_{0}\right) \quad \text { for } \quad n_{0} \leq n<n_{k}, \quad k \in \mathbb{N} .
$$

Since

$$
h\left(n-n_{0}\right) \geq(k-1) \theta \quad \text { for } \quad n_{0} \leq n<n_{k}, \quad k \in \mathbb{N},
$$

from (22) we derive

$$
V(n) \leq \Lambda^{p \frac{h\left(n-n_{0}\right)}{\theta}} V\left(n_{0}\right)=e^{p \frac{\ln \Lambda}{\theta} h\left(n-n_{0}\right)} V\left(n_{0}\right) \quad \text { for } \quad n \geq n_{0} .
$$

This estimate together with (17) and (19) leads to

$$
\sum_{i=1}^{m} \xi_{i} \psi_{i}^{-1} e^{\mu p h\left(n-n_{0}\right)}\left|u_{i}(n)\right|^{p} \leq V(n) \leq e^{p \frac{\ln \Lambda}{\theta} h\left(n-n_{0}\right)} V\left(n_{0}\right)
$$

for $n \geq n_{0}$, from which

$$
\sum_{i=1}^{m}\left|u_{i}(n)\right|^{p} \leq \Omega_{1} e^{-p\left(\mu-\frac{\ln \Lambda}{\theta}\right) h\left(n-n_{0}\right)} \sum_{i=1}^{m} \sup _{\ell \leq n_{0}}\left|\varphi_{i}(\ell)\right|^{p}
$$

for $n \geq n_{0}$, where the constant

$$
\begin{aligned}
\Omega_{1}=\max _{i=\overline{1, m}}\left\{\xi_{i} \psi_{i}^{-1}(h)\right. & +\sum_{j=1}^{m} \xi_{j} \bar{a}_{j}\left|c_{j i}\right| L_{i}^{f} e^{\mu\left(\sigma_{j i}+1\right) h} \sigma_{j i} \\
& \left.+\sum_{j=1}^{m} \xi_{j} \bar{a}_{j}\left|d_{j i}\right| L_{i}^{g} \sum_{\ell=1}^{\infty} \mathcal{K}_{j i}(\ell) e^{\mu(\ell+1) h} \ell\right\} / \min _{i=\overline{1, m}}\left\{\xi_{i} \psi_{i}^{-1}(h)\right\}
\end{aligned}
$$

satisfies $1 \leq \Omega_{1}<\infty$. The statement (14) will follow subsequently and this completes the proof. 


\section{DISCRETE COHEN-GROSSBERG NEURAL NETWORKS}

COROLlaRY 1. Suppose the assumptions and conditions in Theorem 2 are satisfied with (12) replaced by

$$
\begin{aligned}
\underline{a}_{i} \underline{b}_{i} & -\frac{p-1}{p} \sum_{j=1}^{m} \bar{a}_{i}\left|c_{i j}\right| L_{j}^{f}-\frac{p-1}{p} \sum_{j=1}^{m} \bar{a}_{i}\left|d_{i j}\right| \kappa_{i j} L_{j}^{g} \\
& -\frac{1}{p} \sum_{j=1}^{m} \bar{a}_{j}\left|c_{j i}\right| L_{i}^{f}-\frac{1}{p} \sum_{j=1}^{m} \bar{a}_{j}\left|d_{j i}\right| \kappa_{j i} L_{i}^{g}>0, \quad i=\overline{1, m} .
\end{aligned}
$$

Then the impulsive analogue (10) is globally exponentially stable in the sense of (14).

In fact, the inequalities (23) imply that $\Xi_{1}$ defined in (12) becomes an $M$-matrix.

\section{REFERENCES}

[1] AKÇA, H.-ALASSAR, R.-COVACHEV, V.-COVACHEVA, Z.: Discrete counterparts of continuous-time additive Hopfield-type neural networks with impulses, Dynam. Systems Appl. 13 (2004), 77-92.

[2] ARIK, S.-ORMAN, Z.: Global stability analysis of Cohen-Grossberg neural networks with varying delays, Phys. Lett. A 341 (2005), 410-421.

[3] CAO, J.-SONG, Q.: Stability in Cohen-Grossberg-type bidirectional associative memory neural networks with time-varying delays, Nonlinearity 19 (2006), 1601-1617.

[4] CHEN, Z.-RUAN, J.: Global dynamic analysis of general Cohen-Grossberg neural networks with impulse, Chaos Solitons Fractals 32 (2007), 1830-1837.

[5] CHEN, Z.-RUAN, J.: Global stability analysis of impulsive Cohen-Grossberg neural networks with delay, Phys. Lett. A 345 (2005), 101-111.

[6] COHEn, M. A.-GROSSBERG, S.: Absolute stability of global pattern formation and parallel memory storage by competitive neural networks, IEEE Trans. Systems Man Cybernet. 13 (1983), 815-826.

[7] COVACHEV, V.-AKÇA, H.-COVACHEVA, Z.-AL-ZAHRANI, E.: A discrete counterpart of a continuous-time additive Hopfield-type neural networks with impulses in an integral form, Stud. Univ. Žilina Math. Ser. 17 (2003), 11-18.

[8] COVACHEV, V.-AKÇA, H.-YENIÇERIOĞLU, F.: Difference approximations for impulsive differential equations, Appl. Math. Comput. 121 (2001), 383-390.

[9] FIEDLER, M.: Special Matrices and Their Applications in Numerical Mathematics, Martinus Nijhoff Publ., Dordrecht, 1986.

[10] FORTI, M.-TESI, A.: New conditions for global stability of neural networks with application to linear and quadratic programming problems, IEEE Trans. Circuits Systems I Fund. Theory Appl. 42 (1995), 354-366.

[11] GOPALSAMY, K.: Stability of artificial neural networks with impulses, Appl. Math. Comput. 154 (2004), 783-813.

[12] HADAmARD, J.-S.: Sur les Correspondences Ponctuelles, Euvres, Éditions du Centre National de la Recherche Scientifique, Paris, 1968. 


\section{SANNAY MOHAMAD — HAYDAR AKÇA — VALÉRY COVACHEV}

[13] HORN, R. A.-JOHnSON, C. R.: Topics in Matrix Analysis, Cambridge University Press, Cambridge, 1991.

[14] HUANG, Z.-WANG, X.-GAO, F.: The existence and global attractivity of almost periodic sequence solution of discrete-time neural networks, Phys. Lett. A 350 (2006), $182-191$.

[15] LI, Y.: Existence and exponential stability of periodic solution for continuous-time and discrete-time generalized bidirectional neural networks, Electron. J. Differential Equations 32 (2006), 1-21.

[16] LI, Y.: Global exponential stability of BAM neural networks with delays and impulses, Chaos Solitons Fractals 24 (2005), 279-285.

[17] LI, Y.: Global stability and existence of periodic solutions of discrete delayed cellular neural networks, Phys. Lett. A 333 (2004), 51-61.

[18] LIANG, J.-CAO, J.: Exponential stability of continuous-time and discrete-time bidirectional associative memory networks with delays, Chaos Solitons Fractals 22 (2004), $773-785$.

[19] LIANG, J.-CAO, J.-HO, D. W. C.: Discrete-time bidirectional associative memory neural networks with variable delays, Phys. Lett. A 335 (2005), 226-234.

[20] LIANG, J.-CAO, J.-LAM, J. J.: Convergence of discrete-time recurrent neural networks with variable delay, Internat. J. Bif. Chaos Appl. Sci. Engrg. 15 (2005), 581-595.

[21] MOHAMAD, S.: Exponential stability preservation in discrete-time analogues of artificial neural networks with distributed delays, J. Comput. Appl. Math. 215 (2008), 270-287.

[22] MOHAMAD, S.: Computer simulations of exponentially convergent networks with large impulses, Math. Comput. Simulation 77 (2008), 331-344.

[23] MOHAMAD, S.: Global exponential stability in discrete-time analogues of delayed cellular neural networks, J. Difference Equ. Appl. 9 (2003), 559-575.

[24] MOHAMAD, S.: Global exponential stability in continuous-time and discrete-time delayed bidirectional neural networks, Phys. D 159 (2001), 233-251.

[25] MOHAMAD, S.-GOPALSAMY, K.: Dynamics of a class of discrete-time neural networks and their continuous-time counterparts, Math. Comput. Simulation 53 (2000), $1-39$.

[26] MOHAMAD, S.-NAIM, A. G.: Discrete-time analogues of integrodifferential equations modelling bidirectional neural networks, J. Comput. Appl. Math. 138 (2002), 1-20.

[27] SONG, Q.-CAO, J.: Impulsive effects on stability of fuzzy Cohen-Grossberg neural networks with time-varying delays, IEEE Trans. Systems Man Cybernet. Part B 37 (2007), 733-741.

[28] SUN, C.-FENG, C. B.: Discrete-time analogues of integrodifferential equations modeling neural networks, Phys. Lett. A 334 (2005), 180-191.

[29] SUN, C.-FENG, C. B.: Exponential periodicity of continuous-time and discrete-time neural networks with delays, Neural Proc. Lett. 19 (2004), 131-146.

[30] XIANG, H.-YAN, K. M.-WANG, B. Y.: Existence and global stability of periodic solution for delayed discrete high-order Hopfield-type neural networks, Discrete Dyn. Nat. Soc. 3 (2005), No. 3, 281-297.

[31] YANG, F.-ZHANG, C.-WU, D.: Global stability of impulsive BAM type CohenGrossberg neural networks with delays, Appl. Math. Comput. 186 (2007), 932-940. 


\section{DISCRETE COHEN-GROSSBERG NEURAL NETWORKS}

[32] YANG, Z.-XU, D.: Impulsive effects on stability of Cohen-Grossberg neural networks with variable delays, Appl. Math. Comput. 177 (2006), 63-78.

[33] ZHANG, J. Y.-SUDA, Y.-KOMINE, H.: Global exponential stability of Cohen-Grossberg neural networks with variable delays, Phys. Lett. A 338 (2005), 44-50.

[34] ZHOU, T.-LIU, YUEHUA-LIU, YIRONG: Existence and global exponential stability of periodic solution for discrete-time BAM neural networks, Appl. Math. Comput. 182 (2006), 1341-1354.

Received August 30, 2008

\author{
Sannay Mohamad \\ Department of Mathematics \\ Faculty of Science \\ Universiti Brunei Darussalam \\ Gadong BE 1410 \\ BRUNEI DARUSSALAM \\ E-mail: sannay@fos.ubd.edu.bn \\ Haydar Akça \\ Mathematical Sciences Department \\ Faculty of Sciences \\ United Arab Emirates University \\ P. O. Box 17551 \\ Al Ain \\ $U A E$ \\ E-mail: hakca@uaeu.ac.ae \\ Valéry Covachev \\ Department of Mathematics \& Statistics \\ College of Science, P. O. Box 36 \\ Sultan Qaboos University, Muscat 123 \\ SULTANATE OF OMAN \\ Institute of Mathematics \\ Bulgarian Academy of Sciences \\ Sofia \\ BULGARIA \\ E-mail: vcovachev@hotmail.com \\ valery@squ.edu.om
}

